WP 39_12

Lorenzo Cassi

Université Paris 1 Panthéon-Sorbonne, France

Bocconi University, Italy

Lorenzo Zirulia

University of Bologna, Italy

Bocconi University, Italy

The Rimini Centre for Economic Analysis (RCEA), Italy

\title{
FrIENDS AND RIVALS: MODELLING THE SOCIAL RELATIONS OF INVENTORS
}

Copyright belongs to the author. Small sections of the text, not exceeding three paragraphs, can be used provided proper acknowledgement is given.

The Rimini Centre for Economic Analysis (RCEA) was established in March 2007. RCEA is a private, nonprofit organization dedicated to independent research in Applied and Theoretical Economics and related fields. RCEA organizes seminars and workshops, sponsors a general interest journal The Review of Economic Analysis, and organizes a biennial conference: The Rimini Conference in Economics and Finance (RCEF) . The RCEA has a Canadian branch: The Rimini Centre for Economic Analysis in Canada (RCEACanada). Scientific work contributed by the RCEA Scholars is published in the RCEA Working Papers and Professional Report series.

The views expressed in this paper are those of the authors. No responsibility for them should be attributed to the Rimini Centre for Economic Analysis. 


\title{
Friends and Rivals: modelling the social relations of inventors
}

\author{
Lorenzo CASSI \\ CES, Université Paris 1 - Panthéon-Sorbonne, OST, Paris and KITeS, Bocconi University \\ Email : Lorenzo.Cassi@univ-paris1.fr
}

Lorenzo ZIRULIA

University of Bologna, KITeS, Bocconi University and RCEA

Email: lorenzo.zirulia@unibo.it

June 2012

\begin{abstract}
In this chapter we develop a model where a population of inventors is rival in the production of patents. Inventors are embedded in the social space, and this affects the process of knowledge creation and diffusion. Our main contribution to the existing theoretical literature on social networks and knowledge is to introduce explicitly patents and patents' citations. This is interesting per se, and it favours a better comparability of results with those of empirical analysis on the same topic. Results from numerical simulations show that our model is able to replicate the empirical negative relationship between patents' citations and social distance. Furthermore, different social network structures may have an impact on the exact shape of such relationship.
\end{abstract}




\section{INTRODUCTION}

Recently, the role of social networks in the creation and diffusion of knowledge has been intensively studied, both empirically (Giuliani and Bell, 2005; Fleming et al., 2007) and theoretically (Cowan and Jonard, 2004; Cassi and Zirulia, 2008; Morone and Taylor, 2010). The assumption that large part of scientific and technical knowledge is tacit and only partially codifiable, and then face--to--face interactions are required for its transmission leads to the conclusion that social connections typically matter (Powell and Grodal, 2005). In this chapter, we make a contribution to the theoretical literature on the topic. We develop a model where a population of inventors is rival in the production of patents. Inventors are embedded in the social space, and this affects the process of knowledge creation and diffusion. The main element of novelty in our work consists in extending the literature in a favour of a better comparability of results with those of empirical analysis. This is done introducing explicitly in the model the counterpart of proxies for knowledge and knowledge diffusion used in these works: in case of inventors, patents and patent's citations.

Among the different streams of empirical literature on networks and knowledge, our attention in this chapter is focused on studies that investigate the relationship between knowledge flows, measured by patents' citations, and social distance. These works (e.g. Breschi and Lissoni, 2004) find that the probability to observe a citation tie between two patents decrease as the social distance between two patents increase, which is suggestive of the idea that knowledge flows (with decay) through the social network. Our model replicates this evidence and, more importantly, provides new theoretical 
results on the impact of network structure on knowledge flows, in a form that is potentially testable by empirical researchers.

The structure of the chapter is as follows. Section 2 puts our work in the perspective of the existing theoretical literature and creates the link with empirical analysis, thus motivating the extensions we propose. Section 3 describes the model in detail, while Section 4 reports results from numerical simulations. Finally, Section 5 concludes.

\section{THE THEORETICAL AND EMPIRICAL LITERATURE ON SOCIAL NETWORKS AND KNOWLEDGE}

In its formal structure and focus of analysis, this chapter belongs to the theoretical literature on networks and knowledge, initiated by the work of Cowan and Jonard (Cowan and Jonard, 2004). This literature builds on two basic elements. First, it represents the economy as a heterogeneous population of agents involved in the creation and diffusion of knowledge. Second, agents are located on a network, which implies that each agent can "interact" (both in the process of knowledge creation and diffusion) only with a subset of other agents in the society (their "neighbourhood"). Given these commonalities, the existing models differ on several dimensions: the network can be exogenous (Cowan and Jonard, 2003a) or endogenous (Cowan et al., 2003); knowledge has been represented in different ways, as a stock (Morone and Taylor, 2004), as vector of real positive scalars (Cowan and Jonard, 2004), or as a pair constituted by a scalar and an angle (Cowan et al., 2003); different diffusion processes (pair--wise barter, as in Cowan and Jonard, 2004; broadcasting, as in Cowan and 
Jonard, 2003a) and knowledge creation processes (recombination by the receiver, as in Cowan and Jonard, 2003a; individual learning, as in Cowan et al., 2004); further details has been added to characterize the process of knowledge creation and diffusion in specific institutional contests, like the scientific community (Cowan and Jonard, 2003b). The most recent contributions (e.g. Baum et al., 2010, Morone and Taylor, 2010) focused on endogenous network formation of "agents" that are interpreted as firms, thus relating to, and trying to rationalize the results of the vast literature on interfirm technological networks (Zirulia, 2009). In this chapter we take a different route, by making a number of contributions to the current state of the literature that are empirically motivated by the specific context we have in mind, i.e. a population of inventors engaged in the production of patents. We believe that the modifications we propose are both theoretically interesting per se, and useful in bridging the gap with the empirical literature on this issue. First, we consider the role of social networks as an option. This has already been done in the model of Cassi and Zirulia (2008). The idea is that, while agents are embedded in a social network, the actual use of the network is an (economic) choice. Agents will have typically other options for reaching their goal (in this case, learning and consequently producing new patents), rather than interacting socially. Quite obviously, agents will have the possibility of learning individually: so it could be possible that the structure of the network affects its use.

Second, we relax the assumptions of non rivalry among agents. If agents seek knowledge not for its own sake, but to produce "objects" that embodies such knowledge, agents will typically care about knowledge of other individuals, since collectively they participate to a number of "races". This is true for inventors, but also for scientists engaged in the production of papers. These considerations imply, as 
described in detail in the next section, that we will model the problem of choice between learning modes, and we will do that by endowing agents with a simple rule of thumb that implicitly takes into consideration the rivalry among them.

A third, more significant extension concerns the representation of knowledge. Under this aspect, our attempt to create connections with the empirical literature is explicit. There is now a significant amount of empirical work on the structure and role of social networks in "knowledge--intensive" communities, both in the realm of Open Science (Newman, 2000), and in the realm of Proprietary Technology (Balconi et al., 2004; Breschi and Lissoni, 2004, Agrawal et al., 2006). The assumptions that motivate such an empirical program of research are indeed similar to the assumptions that lie at the core of the theoretical literature. Since technical and scientific knowledge is largely "tacit" and "non--codifiable", face--to--face interactions, and consequently social networks, matter for knowledge diffusion and creation. In that respect, the empirical and theoretical literature are largely complementary: at both levels, for instance, it is possible to study how the specific characteristics of a technical or scientific field impact on the emergent properties of a social network structure; or the relation occurring between distances in the social and "cognitive" space. However, the empirical approach needs to tackle the issue of "non--observability" of knowledge, and, especially in large groups, the difficulties in measuring social networks among inventors and scientists. This leads to the question of proxies.

The natural solution to these problems has been to use the "objects" in which knowledge is embodied. In the case of inventors, these are given by patents (Griliches, 1990). Patents are clearly correlated with knowledge possessed by inventors; there is a large availability of data; patents' citations can be used to track "knowledge" flows 
(Jaffe et al., 1993) among individuals and consequently to locate an individual in the "cognitive" space. Furthermore, patent data can be used also to map the social network of inventors, using co--patenting to infer the existence of a social link among the inventors in the research team (e.g. Breschi and Lissoni, 2004).

Among the different streams of empirical literature on the broad theme of knowledge and networks, we focus on a recent set of empirical analysis which has used patent and patent citations in order to investigate the relation between social relations and knowledge flows.

The starting point of these works is to understand better the mechanisms underlying knowledge flows and why knowledge spillovers are geographically bounded. All the articles use a refinement of the methodology originally proposed by Jaffe, Trajtenberg and Henderson (1993). In this seminal article, the three authors considered patent citations as possible paper trails left by knowledge spillovers. Accordingly, they set up an original test to show that citations to prior art tend to come disproportionally from the same geographic area as the cited patents. In order to separate the spillovers effect from other agglomeration effects, they define a control sample of patents similar to the citing ones in terms of technological contains and filled in the same period but not reporting the same reference to prior art. In other terms, the control sample represents the potential citations. Comparing the localisations of the pairs citing--cited patents with the control--cited ones allows in principle to isolate the knowledge spillovers effect from other effects, as, for instance, agglomeration.

While Jaffe, Trajtenberg and Henderson (1993) applied this methodology considering the geographic localisation of inventors, Breschi and Lissoni (2005) applied it considering the social space defined by the network of inventors and find the 
association between patent citations and geographic co--location to be greater for socially connected inventors than others. In other words, Breschi and Lissoni show that geographical proximity matters for knowledge spillovers because knowledge flows through social networks, and social networks are for the most part geographically bounded. In addition to that, Agrawal et al. (2006) show that patent from inventors who move from one region to another continue to be cited by their previous colleagues, reflecting that ties from past collaborations facilitate knowledge flows across regions. Extending this line or reasoning, Breschi and Lissoni (2004), Cassi (2004) and Singh (2005) have investigated the relationship between knowledge flows and social distance. In particular, these authors have show that the probability to observe a citation tie between two patents decrease as the social distance between two patents increase, where the social distance between two patents has been defined as the minimum distance among all the distance between the pair of inventors belonging to the two teams responsible for the patents. ${ }^{1}$ Figure 4.1 (Cassi, 2004) reports the social distance calculated between inventors participating to patents linked by an actual citation (full line) compared to distance between patents linked by a potential citation (dot line), more specifically it compares the frequencies of a citation between two patents (vertical axe - in percent value) relatively to the social distance separating the two (horizontal axe - geodesic distance).

\section{FIGURE 4.1 NEAR HERE}

Citations seem to occur between inventors socially close to each other. The frequencies drop initially very fast: direct connection of being or being at distance 2 seems to be

\footnotetext{
${ }^{1}$ If one inventor has participated in both teams, the social distance is equal to 0 .
} 
important and for distance greater than 4 seems not to have any role. This suggests that knowledge flows through networks are characterized by a fast decay.

In order to create the connection with the empirical literature just described, in our model we will represent knowledge in a way that makes possible, in the same space, also the representation of patents and patents' citations. This makes it possible to produce theoretical results that are directly comparable with empirical ones. In particular, we aim at investigating the determinants of knowledge flows as measured by patents' citations. In that respect, not only we will look at the effect of social distance on the probability of citation, but we also study how different network structures impact on overall intensity of knowledge flows and how the effect of social distance is mediated by the network structure and other characteristics of the system. As the description of the model we will hopefully make clear, in representing patents and citations we try to capture two distinctive features of these objects in reality. First of all, knowledge flows can be measured only if both the "sender" and the "receiver" have obtained an innovation, and then a patent. Second, a citation between two patents does not necessarily entail knowledge flows between the two inventors, since the related patents may be the outcome of autonomous learning.

\section{THE MODEL}

The model considers a population of $N$ economic agents who create and diffuse knowledge over time. Normally we will refer to these economic agents as individual inventors, but one could easily consider them as a team of researchers or firms. In this 
case, some of the assumptions require a different interpretation, which will discuss. The goal of inventors is to transform their knowledge in new patentable ideas, and for that reason the relation among them is characterized by a certain degree of rivalry. Furthermore, inventors are located on a lattice, which is exogenously given and time-invariant, and it identifies the social space. In the process of knowledge creation, inventors can learn in two ways: individually, through introspection or by accessing the piece of codified knowledge embodied in existing patents; socially, by interacting with other inventors in their social neighbourhood.

A clarification is necessary about what we mean with individual and social learning when agents are organisations instead of individual inventors. 'All learning takes place inside individual human heads; an organisation learns in only two ways: (a) by the learning of its members, or (b) by ingesting new members who have knowledge the organisation didn't previously have' (Simon, 1991, p.125). Since in our model the social structure is given, the Simon's option $b$ (e.g. hiring someone) is excluded by assumption. Therefore, the learning organisation is reduced to the learning of its member (i.e. Simon's option $a$ ). According to this, an organisation learns individually if some of its members learn and this could likely occur by members' interaction. This could be thought of as intra--firm learning phenomena. Otherwise, organisations learn socially when (at least) two individuals belonging to two different organisations learn interacting with each other.

This section is structured as follows: first, we describe how knowledge is represented in our model and, given this representation, how we characterize patents and patent's citations. Then, we discuss the options agents have for learning, and the rule of thumb 
we assume they adopt in choosing between them. Finally, we describe the structure of social networks we will consider in our numerical exercises.

\subsection{Knowledge representation, patents and patent's citations}

A primary contribution of the chapter lies in the representation of knowledge. The rationale for developing such a representation is twofold. First, we intend to go beyond a scalar or vector representation of knowledge. These formal representations are simple, and very often convenient in a number of applications: however, they fail in capturing some of the notions put forth appreciatively in the economics of knowledge, and then it is meaningful per se trying to improve upon them. Second, the representation we propose is functional to introduce explicitly the role of patents and patents' citations. The key notion in our model is that of knowledge space. The knowledge space is given by a regular bi--dimensional lattice (i.e. a rectangular grid of cells). Formally, it is denoted as $K \subset N^{2}$. Consequently, it is possible to identify the single cell with a pair of natural number $\left(k_{1}, k_{2}\right) \in N^{2}$.

In each period $t$, each agent is characterized by a knowledge set $K_{i}^{t} \subseteq K$. Graphically, the knowledge possessed by each individual can be represented by cells that are 'turned on' in the grid of knowledge space. In Figure 4.2, the collections of dark cells are an example of knowledge set for an individual.

\section{FIGURE 4.2 NEAR HERE}

Patents are defined as subset of the knowledge space as well. A patentable idea is defined as a squared subset of cells in the knowledge set, whose dimension exceeds a 
lower bound $l$. We denote a patent as $P_{j} \subseteq K$ and with $P$ the set of all conceivable patents. An inventor "produces" a patentable idea at time $t$ whenever such a subset is entirely included in $K_{i}^{t}$. For a patentable idea to become a patent, a novelty criterion must be satisfied. Define $K_{p}^{t}$ as the set of existing patents at time $t$. The novelty criterion requires that $\frac{\left|K_{p}^{t} \cap P_{j}\right|}{\left|P_{j}\right|}<x$, that is that the number of cells belonging to the intersection of $K_{p}^{t}$ and $P_{j}$ cannot exceed a certain percentage $x$ of the cardinality of $P_{j}$. In this set-up, we can quite naturally define relations within the set of patents (i.e. patents citations). A patent $P_{1}$ must cite a pre-existing patent $P_{2}$ whenever $P_{1} \cap P_{2} \neq\{0\}$. Citations, as we will see, can be used to introduce the notion of distance in the patent space. In turn, distance in the space of patents can be used to proxy distance among agents in the knowledge space, and to compare it with distance in the "social" space.

Summarizing, in each period $t$, we need to keep track of $N+1$ knowledge sets: one for each agent, representing their private knowledge, and one set of "social knowledge" corresponding to the set of patents $K_{p}^{t}$. We can note how "social" knowledge (Machlup, 1981) will not generally coincide with the sum of knowledge for all individuals. Then, it can be studied the ability of the system to "transform" private knowledge in social knowledge.

\subsection{Learning}


Learning occurs at time $t$ whenever $K_{i}^{t-1} \subset K_{i}^{t}$. Learning is modelled as the outcome of a random mechanism which gives the probability that a cell $\left(k_{1}, k_{2}\right)$ that does not belong to $K_{i}^{t-1}$ will belong to $K_{i}^{t}$. We assume that learning can occur in two ways: individually, through introspection or by accessing the piece of codified knowledge embodied in existing patents; socially, by interacting with other inventors in their social neighbourhood. Furthermore, we assume no "reduction" in knowledge, in the sense that $\left(k_{1}, k_{2}\right) \in K_{i}^{t-1} \Rightarrow\left(k_{1}, k_{2}\right) \in K_{i}^{t}$.

The forms of learning we have in mind have in common the property of being local. In our context, learning is local in the sense that the probability an agent learns in a specific area depends solely on the interaction with a knowledge set that include areas close to that area (according to the metric implicitly defined in the knowledge space); the knowledge set can be the knowledge set of that agent, or of the agent it is interacting with, or the set of social knowledge.

If learning occurs individually for agent $i$, two are the inputs in its learning process: its existing knowledge set $K_{i}^{t-1}$ and the set of "social knowledge" $K_{P}^{t-1}$. Define as $n_{i}\left(k_{1}, k_{2}\right)$ the number of cells in the Moore neighbourhood of $\left(k_{1}, k_{2}\right)$ that belong to $K_{i}^{t-1}$. Similarly, define as $n_{p}\left(k_{1}, k_{2}\right)$ the number of cells in the Moore neighbourhood of $\left(k_{1}, k_{2}\right)$ that belong to the intersection of $K_{p}^{t-1}$ and $K / K_{i}^{t-1}$, plus $\left(k_{1}, k_{2}\right)$ if it belongs to $K_{p}^{t-1}$.

Then, the probabilistic mechanism of learning is specified as follows:

$$
\operatorname{Pr}_{\text {ind }}\left(\left(k_{1}, k_{2}\right) \in K_{t}^{t} \mid\left(k_{1}, k_{2}\right) \notin K_{i}^{t-1}\right)=q^{*}\left(1-e^{-\lambda\left[n_{i}\left(k_{1}, k_{2}\right)+r n_{p}\left(k_{1}, k_{2}\right)\right]}\right) \quad \forall\left(k_{1}, k_{2}\right) \notin K_{i}^{t-1}
$$


with $0<q<1,0<r<1$ and $\lambda>0$. The assumption $r<1$ captures the idea that agents have only an imperfect ability of absorbing the piece of codified knowledge that is embodied in patents.

Concerning now social learning, assume that agent $i$ and $j$ interact. Define as $n^{a b s}\left(k_{1}, k_{2}\right)$ the number of cells of the Moore neighbourhood of $\left(k_{1}, k_{2}\right)$ that belong to the intersection of $K_{i}^{t-1}$ and $K_{j}^{t-1}$, and $n_{j}\left(k_{1}, k_{2}\right)$ the number of cells in the intersection of the Moore neighbourhood of $\left(k_{1}, k_{2}\right)$ belonging to the intersection of $K / K_{i}^{t-1}$ and $K_{j}^{t-1}$, plus $\left(k_{1}, k_{2}\right)$ if it belongs to $K_{j}^{t-1}$.

Formally, the probabilistic mechanism of learning is specified as follows:

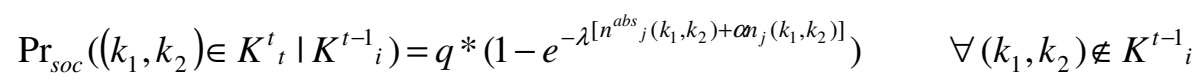

with $0<\alpha<1 . \alpha<1$ captures the role of absorptive capacity (Cohen and Levinthal, 1989): cells that do not belong to the knowledge set of agent $i$ have a smaller impact in increasing the probability of learning.

We need to comment upon the nature of the social learning mechanism we have in mind. The crucial assumption is that any social interaction creates learning opportunities for both agents: the process is bi--directional in nature. In general, the process will be asymmetric, both $e x$--ante (in terms of probability of learning) and $e x-$ post (in terms of actual learning). We believe such an assumption is indeed not restrictive. In abstract terms, it is consistent with the view that any form of interaction 
between two "knowledge--carrying" agents can possibly lead to a process of knowledge creation for each of them.

\subsection{Choosing the learning mechanisms and the dynamics of the system}

Until now, we have described the learning options that are available to the individuals. We need to specify now the criterion agents use to choose between these options. In order to do that, we start describing how the system evolves over time.

Each period, two agents that are connected in the social network are drawn with uniform probability. In other words, we extract one link among those existing in the social network. These two agents are the only two that are allowed to learn at period $t$. We assume that individual learning and social learning are mutually exclusive: agents must choose between individual learning and social learning. Furthermore, we assume that social learning occurs only when both agents are willing to interact socially. In principle, agents should compare the gain from each form of learning and pick up the best option. This is what is done in the simplified framework of Cassi and Zirulia (2008). In this model, however, things are more complex. Assuming that agents have a perfect foresight of benefits from each of the two forms of learning is quite demanding. It implies perfect information about the knowledge set of the involved agents (in particular, about the knowledge set of other agents) and a perfect understanding of the learning mechanisms. Furthermore, we need to take into account the fact that agents are rivals in the production of patents, and then they should consider the effects of their decisions on the probability of learning (and patenting) by other agents, for their 
negative effect on their probability of patenting. Taking rationality to the limit, agents should not act myopically, but they should evaluate the long run effect of their choices on the more general process of knowledge creation and diffusion.

For those reasons, we take a different route. We assume that agents follow a simple rule of thumb, which embodies some assumptions about the information they possess and captures, although roughly, the incentive agents have in choosing one form of learning or the other. We assume that other agents' knowledge is unobservable, and it is not taken into account in the choice of mode of learning. Instead, we assume that an agent has a fairly good perception of its own knowledge. In particular, we assume that an agent knows how far it is from a new patent.

Individual learning is a more focused form of learning: agents learn around their existing area of expertise. Social learning, instead, is modelled as a strategy of "exploration": through interaction, inventors can acquire knowledge far from their current strengths. When an agent is close to patenting, this means it has a strong expertise in a specific area of the knowledge space: if it engages in individual learning, it will have in general a high probability of learning the pieces of information that are missing to produce the new patent; while, if it engages in social learning, it may happen it will learn far from its current focus.

Notice also that if the potential partner possessed the piece of information that is missing to agent $i$ to produce the patent, the probability that such a partner is also close to patenting will be in general significant. Then, the advantage of getting from the partner the key piece of information that is missing must be compared with the risk of losing a new patent, when the missing piece could be acquired though individual learning. Our assumption is consistent with the argument put forth by von Hippel 
(1987) in his case study of informal know--how exchange in the American mini--mills steel industry. ${ }^{2}$

[...] when "Engineer A" encounters a product or process development problem he finds difficult, he activates his network by calling "Engineer B", an appropriately knowledgeable contact who works for a directly competing (or non--competing) firm, for advice. B makes a judgment as to the competitive value of the information A is requesting. If it seems to him vital to his own firm's competitive position, he will not provide it. However, if it seems useful but not crucial [...] B will answer his request as well he can and/or refer him to other experts of his acquaintance.

(Von Hippel, 1987, p. 292).

For these reasons, we assume a simple rule of thumb to drive agents' decision in the use of social network: agents will use the network only if they are both more distant from patenting than a given threshold $w$. Formally, agents will use the network if:

$\forall P_{r} \in P / K_{p}{ }^{t-1}: \frac{\left|P_{r} \cap K_{p}^{t-1}\right|}{\left|P_{r}\right|} \leq x,\left|P_{r} / K^{t-1}{ }_{i}\right| \leq w$

and they will use individual learning otherwise.

\footnotetext{
${ }^{2}$ Von Hippel himself claims for the applicability of his arguments 'to any situation in which individuals or organisations are involved in a competition where possession of property know-how represents a form of competitive advantage' (Von Hippel, 1987).
} 
As a last remark, notice that the fact agents use the social network only if both agree implies that it can happen that an agent would learn sometimes individually even if they are far from patenting (but the other agent is close). Then, the rule we proposed in fact implies that agents will learn individually for sure if close to patenting, while, if not, the choice of mode is outcome of random event (from the point of view of the agent). After the agents have chosen the mode of learning, learning occurs. If a patent is produced, it is registered at the "patent office" and citations are assigned. In the case the agent can patent a number of mutually exclusive patents, the patent with the highest dimension is chosen. If both agents can file for the patent, the patent is assigned randomly to one of them. Then, the economy moves to the next period.

\subsection{The network structure}

As we said, we assume the social network to be exogenous. Therefore, following Cassi and Zirulia (2008), we assume that inventors are "embedded" in a pre--existing web of social relations. While these relations have not been formed for the purpose of exchanging knowledge, they constitute the opportunities for knowledge exchange if the rule of thumb described in Section 3.3 suggests that it is convenient to do so. Following the current literature which focuses on exogenous networks (Cowan and Jonard, 2003a), we keep network density (i.e. the number of total exiting links) fixed, while we vary its structure along two dimensions: average path length and average cliquishness. Average path length is a global concept, defined as the average number of 
steps separating two randomly chosen agents. Average cliquishness is instead a local concept, measuring the degree of link redundancy in an agent's neighbourhood.

We are going to compare three kinds of structure: "regular networks", "random graphs" and "small world". Figure 4.3 gives a graphical example for each class of networks, for graphs with 16 nodes and 32 edges.

\section{FIGURE 4.3 NEAR HERE}

A regular network is characterized by a high cliquishness and a high average path length, a random network by low cliquishness and low average path length, and the "small world" by an average distance which is close to the average distance of random network, and a cliquishness that is instead closer to that one of regular networks. In their important contribution, Watts and Strogatz (1998) have proposed a model that is able to generate these different structures as a function of a single parameter. Consider a set of agents $I=\{1, \ldots N\}$. For any $i, j$ define the variable $\chi(i, j)$, which takes value 1 if the two agents are connected, and 0 otherwise. Then, the network $G=\{\chi(i, j) ; i, j \in I\}$ corresponds to the list of all pairwise relationships between agents. Then the neighbourhood of agent $i$ is formally defined as $\Gamma_{i}=\{j \in I /\{i\}: \chi(i, j)=1\}$. A path connecting two agents $i$ and $j$ is defined as a set of pairwise relationships $\left\{\left(i, i_{i}\right), \ldots ;\left(i_{k}, j\right)\right\}$ such that $\chi\left(i, i_{i}\right)=\ldots=\chi\left(i_{k}, j\right)=1$; the distance between $i$ and $j d(i, j)$ is given by the number of steps in the shortest path between $i$ and $j$. Given this notation we can define the average path length as $L=\sum_{i, j \in I} \frac{d(i, j)}{(N(N-1) / 2)}$. The cliquishness of a set $S \subseteq I$ is defined as the proportion of pairwise relationships in S over the total number of possible number of relationships, formally $\operatorname{cl}(S)=\frac{\sum_{i, j \in S} \chi(i, j)}{\# S(\# S-1) / 2}$. In the 
model, average cliquishness is given by the average value of $\operatorname{cl}(S)$ for $S=\Gamma_{i}, i \in I$, that is $C=\sum_{i \in I} \frac{c l\left(\Gamma_{i}\right)}{N}$.

Watts and Strogatz consider a family of graphs with a given number of nodes $N$ (the agents) and total number of links among them, $\frac{n^{*} N}{2}$. They start from a ring of $n$ vertices, i.e. a regular periodic lattice with $n$ nearest neighbours ( $n$ even). Loosely speaking, they consider $N$ agents located on a circle, each of them connected (by undirected edges) with the $n$ nearest neighbours.

The next step is to implement the following algorithm: choose a vertex and the edge that connects it to its nearest neighbour in a clockwise sense. With probability $p$, re--connect this edge to a vertex chosen uniformously at random over the entire ring, with duplicate edges forbidden; with probability 1-p, leave the edge in place. The process is repeated by moving clockwise around the ring, considering each vertex in turn until one lap is completed. Next, consider the edges that connect vertices to their second nearest neighbours clockwise. As before, rewire randomly each of these edges with probability $p$, and continue the process, circulating around the ring and proceeding outward to more distant neighbours after each lap, until each edge in the original lattice has been considered once.

Varying $p$, it is possible to build graphs having different values for average path length and average cliquishness, $L(p)$ and $C(p)$. Both $L(p)$ and $C(p)$ are monotonically decreasing in $p$. At one extreme, for $p=0$, we have a regular network, with high average cliquishness and high average path length. At the other extreme, for $p=1$, we have a random network, where both average path length and average cliquishness are low. Indeed, the important contribution by Watts and Strogatz is to show that trade--off 
between the local concept of cliquishness and the global notion of average distance is not so severe as it could appear at first sight. For a non--negligible region of parameter $p$ 's space (approximately, the interval $[0.01 ; 0.1]$ ), they show that $L(p) \approx L(1)$, but $C(p)>>C(1)$. When in a regular network some "shortcuts" are created connecting distant parts of the graphs, average path length is dramatically reduced, but cliquishness is substantially preserved. Watts and Strogatz call this network structure 'small world'. The 'small world' model by Watts and Strogatz is a convenient representation to compare different sociological theories on the structural properties that are conducive to the formation of social capital (Verspagen and Duysters, 2004). On one hand, regular networks, characterized by close triads and dense and cohesive subgroups, may favour the emergence of common rules and language, reduce ex ante informational asymmetries and increase ex post cooperation (Coleman, 1988); on the other hand, random networks, characterized by low levels of links redundancy, may favour the access of diverse sources of information (Burt, 1992). Small world networks, being in some sense intermediate between the two, may guarantee the benefits of both. In our model, we will consider network structures in line with Watts and Strogatz. Assuming that inventors located on a circle, and starting from a regular network, we apply Watts and Strogatz's algorithm for three different values of $p$, generating the three different typologies of networks.

\section{NUMERICAL SIMULATIONS}


In this paragraph we discuss results from numerical simulations. In our exercise, we vary three parameters. ${ }^{3}$ First of all, we consider three values for the parameter $p$ in the rewiring procedure, so that we compare three network structures: regular networks ( $p=0.007)$, "small worlds" $(p=0.07)$ and random networks $(p=0.7)$. Then, we modify two other parameters in order to compare the three network structure under different scenarios. The first parameter is $r$ in Eq. (4.1), which captures the role of patents in individual learning, since it measures the ability to decoding the information represented in a patent. The second parameter we are going to consider is $w$, which determines the use of social network: agents will use the network only if they are both more distant from patenting than a given threshold $w$. It can be interpreted as a degree of competition of the patent race: the higher the parameter value, the harsher the competition between inventors. In the baseline scenario, $r$ is fixed equal to 0.25 and $w$ to 8 . Then, we modify just one parameter at once to allow us to appreciate its effect relatively to what is obtained under the baseline simulation set of parameters, reported in the appendix. In particular, we will consider $r=0.1$ (i.e. the use of social network is a more suitable way to learn than individual learning compared to the baseline scenario).and $w=12$ (i.e. the degree of competition among inventors is higher in this case compared to the baseline scenario). ${ }^{4}$ The following table displays the different

\footnotetext{
${ }^{3}$ The complete set of parameters that are constant across simulations is reported in the Appendix. Furthermore, the knowledge space is given by a square grid of 100 cells. As for the initial conditions, for each agent the probability that a cell $\left(k_{1}, k_{2}\right)$ belong to the initial knowledge set is 0.01 if $30 \leq k_{1}\left(k_{2}\right) \leq 70$, and 0 otherwise.

${ }^{4}$ Moreover, another parameter has been modified in order to check the robustness of results, namely the degree of novelty each patents should be satisfy in order to be granted (i.e. $x$, the fraction of cells not overlapping with other patents). As a further check for robustness, we considered different seeds for the
} 
simulation we run reporting some basic statistics about the number of patents per inventor distribution at the steady state, i.e when the last patent is assigned and therefore patents produced 'occupied' all the knowledge space available (this occurs around period 42,000 in our runs).

\section{TABLE 4.1 NEAR HERE}

Table 4.1 shows that the mean number of patents per inventor, and then their total number, is similar across runs. In particular, the three network structures guarantee an almost equally efficient transformation of the knowledge space into patents.

Furthermore, all the distributions are highly skewed: most inventors have few patents, while a few inventors have a large number of patents. This is what we exactly observe in real data about patents. Given the structure of the model, the result is not surprising: the learning mechanisms we imposed are highly cumulative and naturally generates an unequal distribution of patents.

However, the distribution varies, as measured by the median, the maximum number of patents and by the degree of skewness independently from the values of other parameters. The distribution is more skewed for the random network than for the small world and the regular network (and related to that, the median is lower and the maximum is higher). The latter have a more uniform distribution of patents in the left part of the distribution, and a smaller number of patents in the right extreme. Notice that there are reasons why one should be concerned with equity issues: if inventors are firms, unequal distribution of patents can negatively affect concentration in industries, and through it, social welfare.

generation of random numbers. The results are qualitatively unchanged. Further information are available on request. 
Figure 4.4 reports the number of patents granted over time as a function of different network structures, for each scenario. For the sake of readability, random network has been considered as a benchmark. For instance the cumulated number of patents produced under the small world structure has been divided by the number of those produced by the random network and then rescaled to zero, i.e. the level of production of random network corresponds to abscissa axe. Negative value means the production of small world is smaller than random network and vice versa.

\section{FIGURE 4.4 NEAR HERE}

While the behaviour of the total number of patents is very similar across the three network structures, some divergence seem to merge in the process towards steady state (especially between period 20,000 and 32,000) where the random network produce a greater number of patents. This seems particularly true when the capacity of decoding patents is smaller (i.e. $r=0.1$, the second plot reported in Figure 4.4).

As we discussed in Section 2, the primary aim of our paper is to compare our theoretical results with the empirical analysis on the relationship between social distance and the probability of citations. The first remark to make is that we cannot replicate exactly the same exercise. Indeed, the empirical procedure, now standard in the literature, requires building a set of control patents. These are patents in the same technological class and with the same application date of the citing patent, but that did not cite the cited patent (refer to the same prior art). Informally, these are defined as patents that could have cited a patent, but that did not. Control patents cannot exist in our model: the probability that a patent cites another patent if it should is 1 . For this reason we have to take a different route. In the following figures, to assess the role of social distance in affecting citations (and then knowledge diffusion) we will report the average number of citations 
per pair of agents, according to the social distance between inventors. In order to make the different network structure comparable independently on the number of citation effectively reported, we report relative measure: average number of citations for a given social distance by the overall average number of citations.

In our model, assessing the role of network structure in affecting the process of knowledge creation and diffusion, as captured by relations of citations across patents, is equivalent to assess the role of two properties of networks, namely cliquishness and average distance.

Ceteris paribus, both an increase in cliquishness and a decrease in average distance increase knowledge diffusion. Low average distance positively affects knowledge diffusion because it gives access in few steps to knowledge created elsewhere in the network. An important remark is needed, however. Since social learning occurs stochastically, but with a positive probability, the expected amount of time for a piece of knowledge to flow from an agent to another decreases with social distance, but the probability of learning tends to 1 as time elapses. ${ }^{5}$ Nevertheless, expected time matters, when knowledge flows are captured by patent citations. If knowledge flows slowly from $i$ to $j$, there is a low probability that agent $j$ can "invent around" this piece of knowledge and file a patent (then making knowledge flows visible) before some other agent, in particular agent $i$, anticipates it in the "patent race". High cliquishness positively affects knowledge diffusion, too. Suppose we take two agents $i$ and $j$, such that $j$ possesses the piece of knowledge that $i$ does not have. Notice

\footnotetext{
${ }^{5}$ In Cowan and Jonard (2004) and Cassi and Zirulia (2008) low average distance matters for diffusion even in the long run because of dispersion in each passage.
} 
that a direct link between $i$ and $j$ does not guarantee that the piece of knowledge will flow from $i$ to $j$. First, we modelled learning as a stochastic process: when agent $i$ and $j$ interact, $i$ learns only with some probability. Second, there is "strategic effect", which is related to the requirement of double coincidence of wants: when the pair $i j$ is selected, agent $i$ could refuse to interact with $j$.

A cliquish network has the property that there are multiple, short paths linking two connected agents $i$ and $j$. This increases the probability that the piece of knowledge possessed by agent $j$ flows to agent $i$ for two reasons. First, there is a probability that knowledge flows from $i$ to $j$ indirectly through some alternative short path linking $i$ and $j$. Second, the probability that $i$ learns directly from $j$ increases. This is due to the local nature of learning. For sake of concreteness, consider an agent $k$ which is a common acquaintance of $i$ and $j . j$ 's piece of knowledge can lead $k$ to learn another piece of knowledge which is close to the previous one in the knowledge space. If this piece of knowledge is "transmitted" to $i$, this increases the probability that $i$ learns from $j$, due to the role of absorptive capacity (Eq. (4.2)). Notice that the mechanisms just described do not involve the sources of benefits (common language, reputation enhancement, information diffusion) normally attributed to "cliquish" networks. Figure 4.5 reports the average number of citations as a function of social distance, in relative terms. The value of average citation is however reported in the legend of the graph.

\section{FIGURE 4.5 NEAR HERE}

The figures are noteworthy for two reasons: first, independently from the network structure, the average number of citations decreases with social distance, consistent the empirical evidence; second, the structure of the network affects such a relationship. 
The negative relationship between citations and social distance derives quite directly from the hypothesis that knowledge possessed by inventors but not embodied in patents can be accessed only through face--to--face interaction. This increases the probability that two agents close in the social space invent in close area of knowledge space, and then cite each other.

With respect to network structure, it turns out that, for the parameterization of the baseline simulation, clustering is the most important property favouring knowledge diffusion. The regular network and the small world dominate, in terms of average number of citations, the random network.

Somewhat paradoxically, the random network, although it reduces the average distance among inventors, is the worst structure for knowledge diffusion, because of its lack of multiple paths among inventors. Also notice that is the lower rate of knowledge diffusion that generates the more unequal distribution of patents: knowledge diffusions tend to mitigate the effect of the cumulativeness of learning. In the parameterization of the baseline simulation, we consider $r=0.25$. Notice that access to patents in individual learning can be considered as imperfect substitute for "shortcuts" in the network, because it allows the access to knowledge created "far" in the social space (imperfection is due to the fact that $r<1$ and access is limited to patented knowledge). One could expect that reducing $r$ should increase the importance of social connections to distant area in the network, and then favour small world and random networks over regular networks. The second plot of Figure 4.5 shows that this is actually the case.

From the graph, we notice that for a low value of $r$, the small world, which has both low average distance and high clustering, maximizes the degree of knowledge diffusion. 
This is true for short social distance: indeed, for $r=0.1$, the links to distant part of the network are crucial to generate variety in knowledge within the clique, and then knowledge diffusion.

In the bottom of Figure 4.5, we display the results when the level of competition is greater (i.e. $w=12$ ). By assumption, in this case, inventors are smaller incentives to learn socially (this is also shown by the lower rates of average citation). As a consequence, the social proximity is less important: being very close is not so determinant. Of course having a direct access to someone (distance equal to 1) is an advantage but apparently it is less important than for other scenarios: it is enough to be connected indirectly to get some gain since the effect of social distance is still positive (i.e. not smaller than 1) until distance 5 for regular network and to a lesser degree for small world as well.

\section{CONCLUSION}

It is now common to look at knowledge as a key input in the growth process of firms and countries. This view is now widely shared not only by economists and other social scientists, but by policy-makers as well (think, for instance, at the so called "Lisbon Agenda"). Moreover, in the process of knowledge creation and diffusion, various forms of collaborations and social interactions, to which we can refer as "networks" in a broad sense, are perceived as having a crucial role.

While both the theoretical and the empirical literatures on these themes have spurred in recent years, the connections between the two has been limited. This chapter tries to fill this gap. In particular, we developed a model of knowledge creation and diffusion in a 
population of inventors, where knowledge is represented in a way that makes possible to consider theoretically the proxies of knowledge and knowledge flows, i.e. patents and patents citations, used in the empirical analysis.

Our analysis was focused on the network determinants of knowledge flows. The empirical analysis suggests a negative relation between social distance and knowledge flows, measured by the probability of citation. First of all, our model is able to reproduce such a negative relation. In addition to that, we produced other results, which constitute testable (but to the best of our knowledge, never tested) implications of our model. In particular, we showed that the relationship between social distance and knowledge flows is affected by structure of the network in which inventors are embedded. Both high clustering and low average distance positively affects knowledge diffusion, but access to the pool of existing patents can be a substitute for connection between otherwise distant parts of the network. Furthermore, we showed that when the incentives to use the social network are lower (in our case, because of higher competition), then social distance is less relevant in explaining patents' citations. implications of our model.

While we believe that the specific point addressed in this chapter is important, our model could provide insights on other issues as well. We intend to explore these avenues for further research in the future. First of all, the model could be enriched by considering the network as endogenous. In this case, the past patents of an inventor could work as signal driving the formation of links, when knowledge is otherwise not observable. Second, we could explore how different interpretations of patenting requirements (e.g. the novelty criterion) can affect the validity of the proxies used in the empirical work. 
Appendix

\section{LIST OF PARAMETERS}

\begin{tabular}{|l|l|l|}
\hline Parameter & Description & $\underline{\text { Value }}$ \\
\hline$N$ & Number of inventors & 100 \\
\hline$n$ & Average number of links & 6 \\
\hline$q$ & per agent & \\
\hline$\alpha$ & Equation 1, 2 & 0.025 \\
\hline$\lambda$ & Equation 2 & 0.5 \\
\hline$x$ & Equation 1,2 & 0.5 \\
\hline$w$ & Novelty criterion & 0.6 \\
\hline$l$ & Equation 3 & 8 \\
\hline$T$ & Minimum size of patents & 5 \\
\hline & (side) & 50000 \\
\hline & Number of periods & \\
\hline & & \\
\hline & & \\
\hline & & \\
\hline & & \\
\hline & & \\
\hline
\end{tabular}




\section{REFERENCES}

Agrawal, A., Cockburn, I. and McHale, J. (2006) 'Gone but not forgotten: knowledge flows, labour mobility and enduring social relationships', Journal of Economic Geography, 6 (5): 571-91.

Balconi, M., Breschi S. and Lissoni, F. (2004) 'Networks of inventors and the role of academia: an exploration of Italian patent data', Research Policy, 33 (1):127-45.

Baum, J., Cowan, R. and Jonard, N. (2010) 'Network--independent partner selection and the evolution of innovation networks', Management Science, 56 (11): 2094-2110. Breschi, S. and Lissoni, F. (2004) 'Knowledge networks from patent data: Methodological issues and research targets', in W. Glänzel, H. Moed, U. Schmoch (eds) Handbook of Quantitative S\&T Research, Dordrecht: Kluwer Academic Publishers. Breschi, S. and Lissoni, F. (2005) 'Cross--firm inventors and social networks: localised knowledge spillovers revisited', Annales d'Economie e de Statistique, Issue 79/80: 189209.

Burt, R.S. (1992) Structural Holes: the Social Structure of Competition, Cambridge: Harvard University Press.

Cassi, L. (2004) Conoscenza e Reti Sociali: tre saggi, PhD Dissertation, Ancona University.

Cassi, L. and Zirulia L. (2008) 'The opportunity cost of social relations: the effectiveness of small world', Journal of Evolutionary Economics, 18 (1): 77-101. Cohen, W.M. and Levinthal, D.A. (1989) 'Innovation and learning: the two faces of R\&D', Economic Journal, 99 (397): 569-596. 
Coleman, J. (1988) 'Social capital in the creation of human capital'. American Journal of Sociology 94 (Supplement): S95-S120.

Cowan, R., David, P. and Foray, D. (2000) 'The explicit economics of knowledge codification and tacitness', Industrial and Corporate Change, 9 (2): 211-253.

Cowan, R. and Jonard, N. (2003a) 'The dynamics of collective invention', Journal of Economic Behavior and Organization, 52 (4): 513-32.

Cowan, R. and Jonard, N. (2003b) 'The Workings of Scientific Communities' in A. Geuna, A.J Salter and W.E. Steinmueller (eds.) Science and Innovation. Rethinking the rationales for funding and governance, Cheltenham: Edward Elgar.

Cowan, R. and Jonard, N. (2004) 'Network structure and the diffusion of knowledge', Journal of Economic Dynamics and Control, 28 (8): 1557-75.

Cowan, R., Jonard, N. and Ozman, M. (2004) 'Knowledge dynamics in a network industry', Technological Forecasting and Social Change, 71(5): 469-84.

Cowan, R., Jonard, N. and Zimmermann, J.B. (2002) 'The joint dynamics of networks and knowledge', in R. Cowan and N. Jonard (eds.) Heterogeneous Agents and Interactions and Economic Performance, vol. 521, Lecture Notes in Economics and Mathematical Systems, Berlin: Springer.

Cowan, R., Jonard, N. and Zimmermann, J.B. (2003) 'On the Creation of Networks and Knowledge' in M. Marsili and A.P. Kirman, (eds.) Proceedings of the $8^{\text {th }}$ WEHIA Conference on Heterogeneous Interacting Agents, Berlin: Springer.

Fleming, L., King, C. and Jud, A.I. (2007) 'Small worlds and regional innovation’, Organization Science, 18 (6): 938-54.

Giuliani, E. and Bell, M. (2005) 'The micro--determinants of meso--level learning and innovation: evidence from a Chilean wine cluster', Research Policy, 34(1): 47-68. 
Griliches, Z. (1990) 'Patent statistic as economic indicators: a survey', Journal of Economic Literature, 28 (4): 1661-707.

Jaffe, A.B., Trajtenberg, M. and Henderson, R. (1993) 'Geographic localisation of knowledge spillover as evidenced by patent citations', The Quarterly Journal of Economics, 108 (3): 577-98.

Machlup, F. (1980) Knowledge: Its Creation, Distribution and Economic Significance, Volume I: Knowledge and knowledge production. Princeton: Princeton University Press.

Morone, P. and Taylor R. (2004) 'Knowledge diffusion dynamics and network properties of face to face interactions', Journal of Evolutionary Economics, 14 (3): 327 51.

Morone, P. and Taylor, R. (2010) Knowledge Diffusion and Innovation: Modelling complex entrepreneurial behaviours, Cheltenham: Edward Elgar Publishing. Newman, M.E.J. (2000) 'Who is the best connected scientists? A study of scientific coauthorship networks', SFI working paper 00-12-64.

Powell, W.W. (1990) 'Neither market nor hierarchies: network form of organisations', Research in Organisational Behaviour, 12: 295-336.

Powell, W.W. and Grodal, S. (2005) 'Networks of innovators', in J. Fagerberg, D. Mowery and R. Nelson (eds.) The Oxford Handbook of Innovation, Oxford: Oxford University Press.

Simon, H.A. (1991) 'Bounded rationality and organizational learning', Organisation Science, 2 (1): 125-34.

Singh, J. (2005) 'Collaborative network as determinants of knowledge diffusion patterns', Management Science, 51 (5): 756-70. 
Verspagen, B. and Duysters, G. (2004) 'The small worlds of strategic technology alliances', Technovation, 24 (7): 563-71.

von Hippel, E. (1987) ‘Cooperation between rivals: informal know--how trading’, Research Policy, 16 (6): 291-302.

Watts, D. and Strogatz, S.H (1998) 'Collective dynamics of "small world" networks', Nature, 393: 440-42.

Zirulia, L. (2009) 'The dynamics of networks and the evolution of industries: a survey of the empirical literature', in F. Malerba and N. Vonortas (eds.) Innovation Networks in Industries, Cheltenham: Edward Elgar Publishing. 
Table 4.1: Patent per inventor distribution. Different simulation runs

\begin{tabular}{|c|c|c|c|c|c|c|c|}
\hline Simulation run & Network & Average & Median & Std dev & Skewness & Max & Min \\
\hline \multirow{3}{*}{ baseline } & $\begin{array}{l}\text { REGULAR } \\
\text { REG }\end{array}$ & 7,67 & 4,5 & 13,00 & 6,72 & 119 & $\overline{0}$ \\
\hline & SMALL WORLD & 7,72 & 5,5 & 10,68 & 6,56 & 99 & 0 \\
\hline & RANDOM & 7,54 & 2 & 19,61 & 5,85 & 162 & 0 \\
\hline \multirow{3}{*}{$r=1$} & REGULAR & 7,64 & 6 & 7,01 & 2,46 & 46 & 0 \\
\hline & SMALL WORLD & 7,61 & 5 & 10,55 & 4,31 & 81 & 0 \\
\hline & RANDOM & 7,68 & 2 & 21,81 & 5,95 & 167 & 0 \\
\hline \multirow{3}{*}{$w=12$} & REGULAR & 7,51 & 5 & 8,57 & 2,83 & 57 & 0 \\
\hline & SMALL WORLD & 7,54 & 5 & 8,05 & 2,34 & 52 & 0 \\
\hline & RANDOM & 7,54 & 2 & 23,42 & 7,32 & 213 & 0 \\
\hline
\end{tabular}


Figure 4.1: Social distance and citations. Source: Cassi (2004)

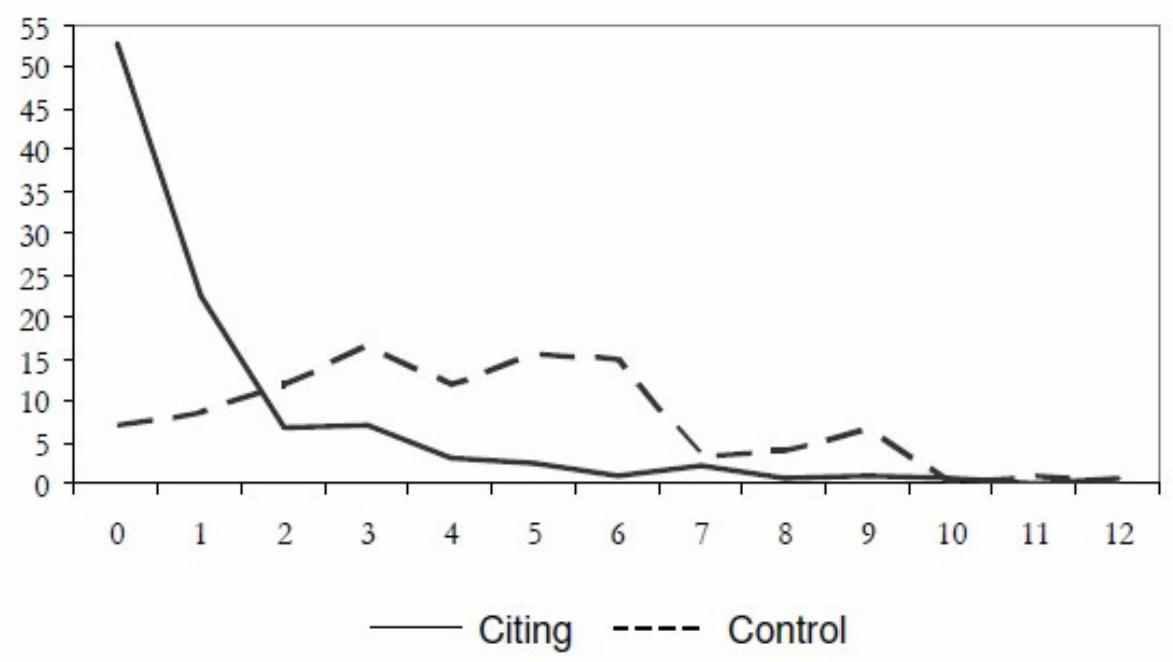


Figure 4.2: An example of knowledge set

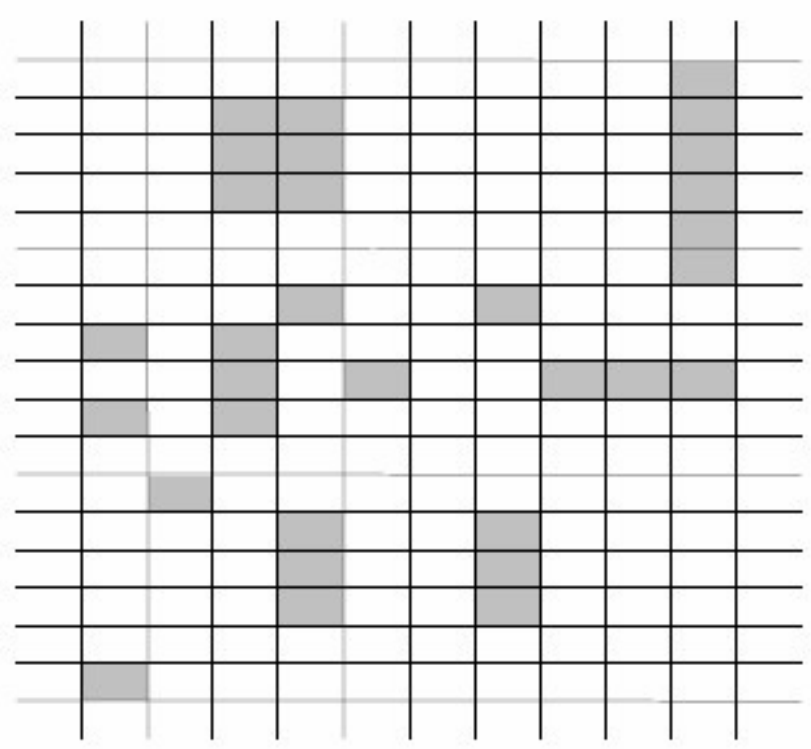


Figure 4.3: The three social structures

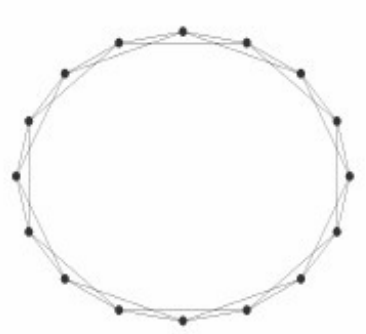

regular network

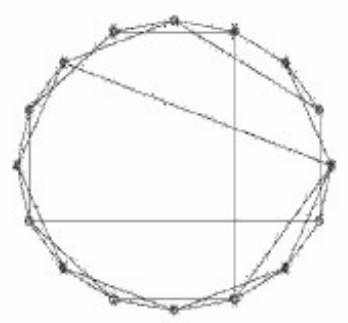

"small world"

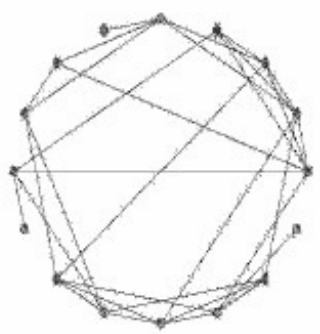

random network 
Figure 4.4: Number of patents over time

Baseline

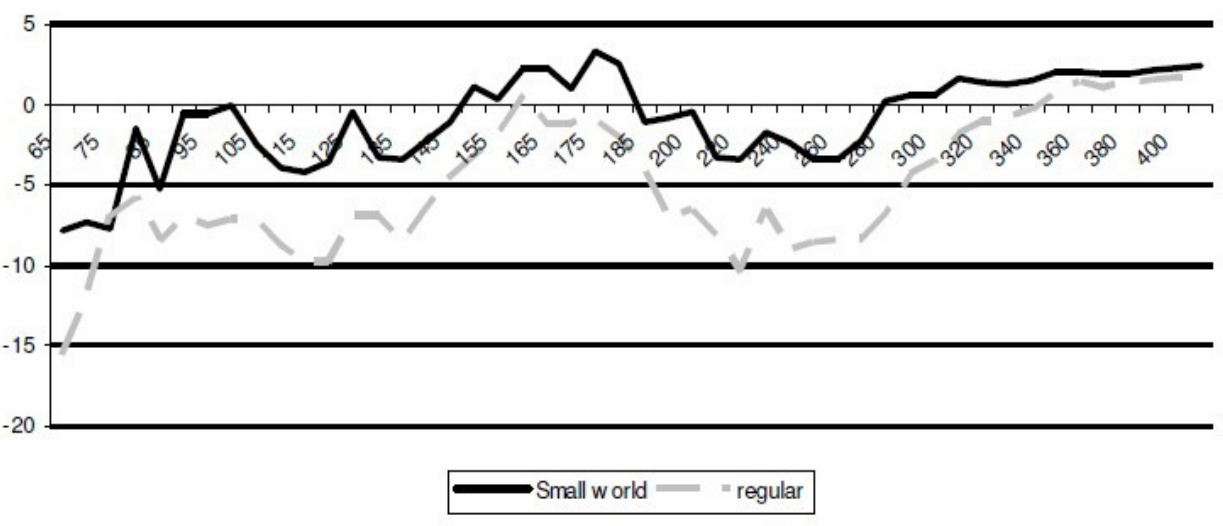

$r=1$

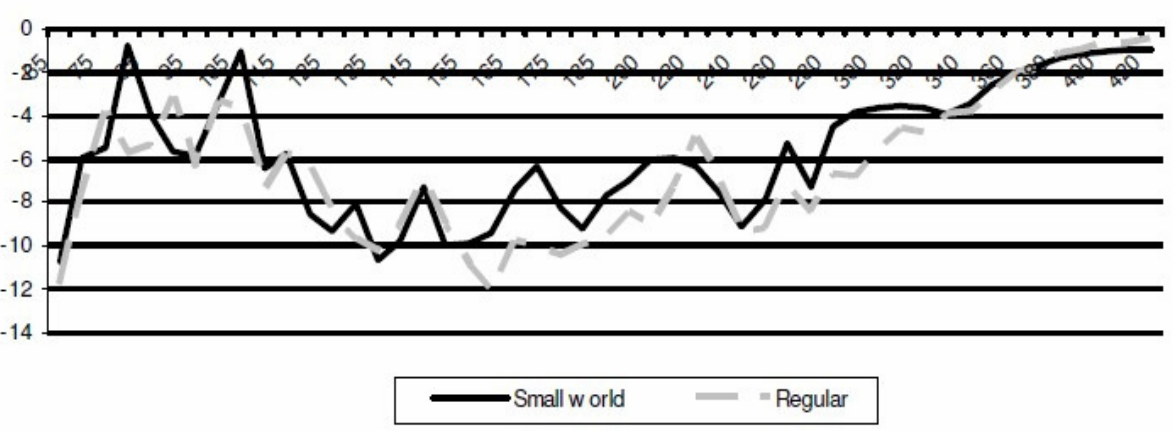

$W=12$

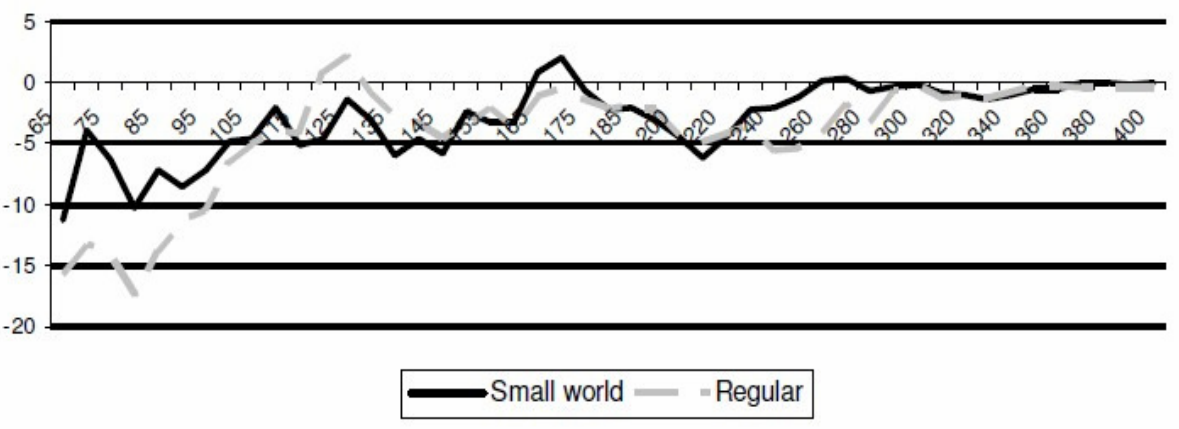


Figure 4.5: Social distance and knowledge diffusion (normalized to average citations)

\section{Baseline}

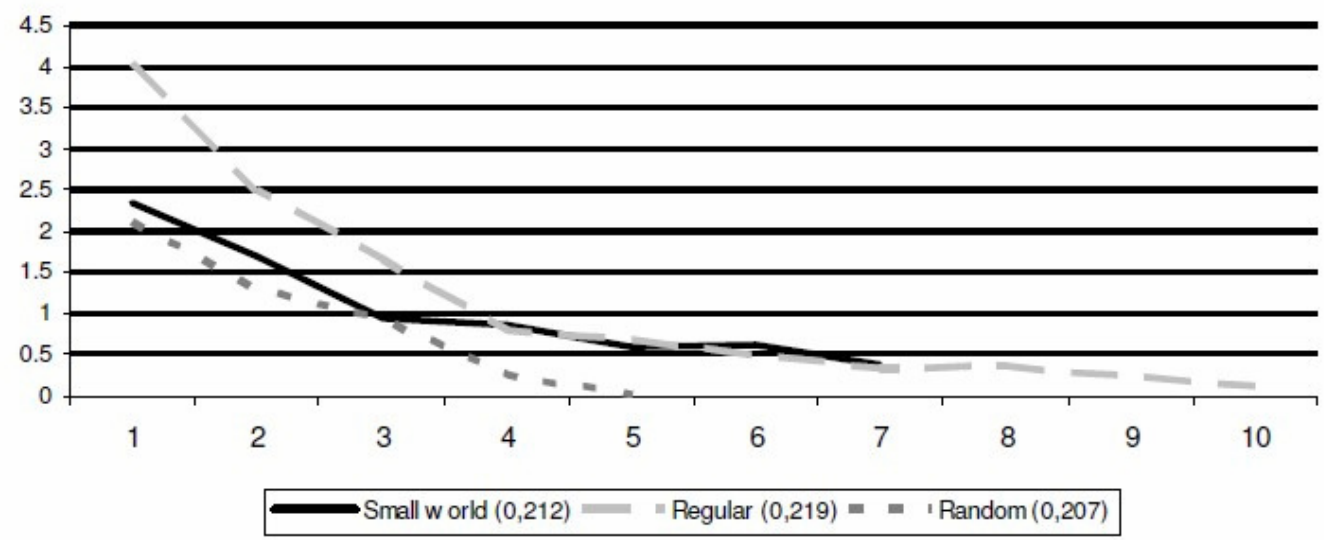

$r=1$

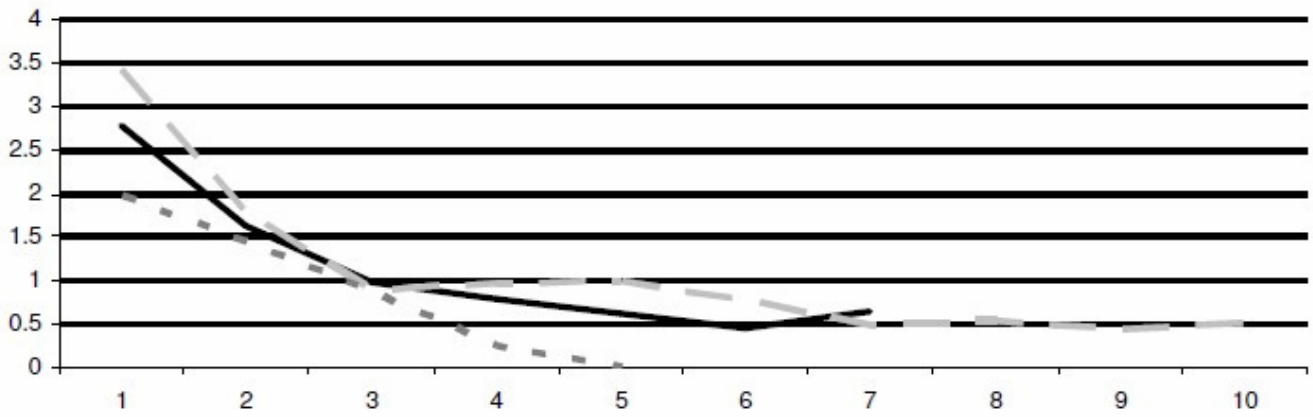

-Small w orld $(0,218)=$ = Regular $(0,214)=$ = ' Random $(0,22)$

$W=12$

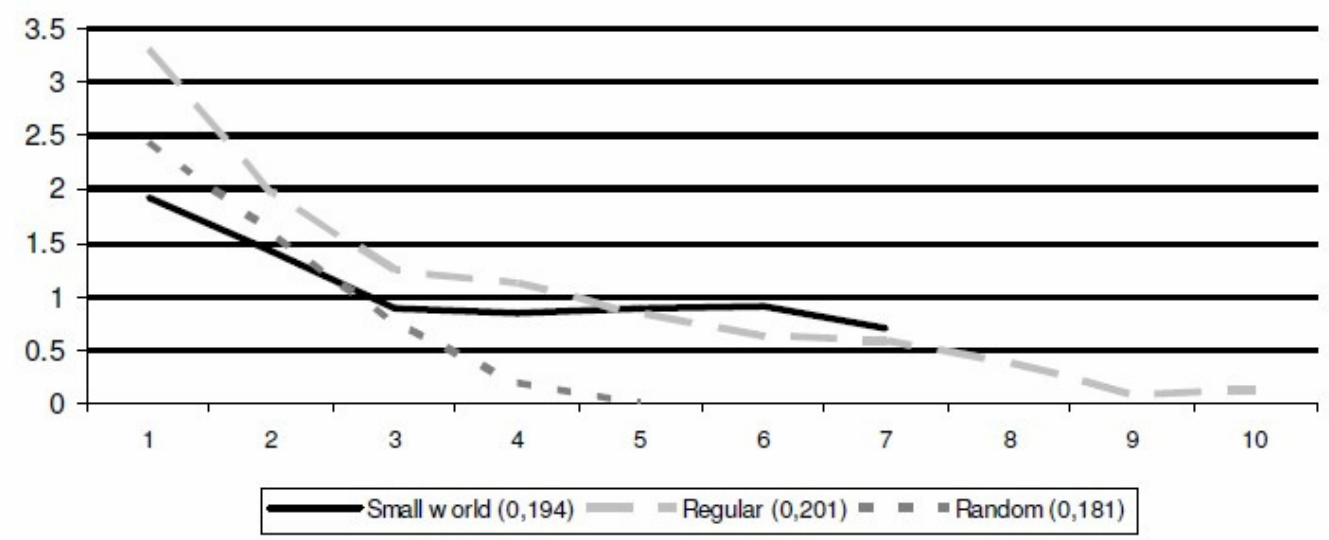

